\title{
Log Data Analysis of Player Behavior in Tangram Puzzle Learning Game
}

\author{
https://doi.org/10.3991/ijim.v12i8.9280 \\ Ivenulut Rizki Diaz Renavitasari ${ }^{(凶)}$, Ahmad Afif Supianto, Herman Tolle \\ Brawijaya University, Malang, Indonesia \\ ivenulutrizki@gmail.com
}

\begin{abstract}
Understanding player behavior in a game is quite important to know people model thinking. Through puzzle games, people can train spatial abilities in a geometry lesson. Spatial ability consists of five groups, one of them is perceptual speed. The Perceptual Speed factor is characterized by the speed in finding a configuration in a mess of distracting material. This research purposes to know user behavior in Tangram Puzzle game based on user data log. Analysis user behavior from two aspects: the dominant of first selecting a puzzle pieces and the correlation between the number of puzzle completion with time. And based on this research can be concluded that player will choose a shape that easily to identified and has a larger size than other to be completed first. Whereas the correlation between the number of completion and time is on a moderate level and quite significant.
\end{abstract}

Keywords - user behavior, log data, tangram puzzle

\section{Introduction}

According to the results of the study of Trend in International Mathematics and Science Study (TMISS) 2015, in general Indonesia is weak in all aspects of content and cognitive, either math or science [1]. Indonesia got a math score of 397 points with a ranking 45 of 50 countries. On geometry lesson, Indonesian students got an average below International students with an average grade of 28 [2]. Geometry is a mathematical science that studies the forms of a two-dimensional figure. Through geometry, it can help people to visualize, spatial reasoning, and modeling to solve problems [3]. This is relevant in the research [3], [4], [5] that to solve the problem of geometry required good spatial ability. Spatial ability is the ability to manipulate visual patterns [4]. On the research [6], Carroll (1993) detected five major clusters of spatial ability: Visualization, Spatial Relations, Closure Speed, Flexibility of Closure, and Perceptual Speed. The perceptual Speed factor is characterized by the speed in finding a given configuration in a mess of distracting material.

Currently, multimedia development is growing rapidly in Indonesia. Multimedia can be utilized in various area and one of them to the learning process and training people ability. An educational game is one of the utilization of multimedia. In addition, to relieving stress, the game can be used learning system while playing. One of the most 
important roles of information technology in education is to design new learning activities [7]. To improve spatial ability to solve a geometry problem, therefore, needed media that can be used to assist in visualizing form. Tangram Puzzle is known to be very effective in teaching and assessing geometric and spatial reasoning abilities of various age [5]. In completing a puzzle, people must be able to identify, imagine and analyze the puzzle pieces. Playing a puzzle will challenge somebody finding out how to solve the puzzle and how fast the solution will be. Every strategy used will train the mind to work efficiently in new and different ways. When matching form, it trains spatial abilities that demand physical and mental activity. Because we will imagine a picture and layout in mind when playing the puzzle. At that time ability of brain will develop and able to create a picture in mind, so make imaginative and creative. we learn reversal, displacement, and rotation from move the tangram's pieces to make a right geometry shape. This provides a real picture for those whose learning orientation in visual through.

The definitions of user behavior are various in different areas. In the field of mobile device research, the user behavior can be concreted to user data collected from mobile phone users when they use services provided by mobile phones. Those data can be used to reflect the user behavior in turn. Relevant research is conducted by [8] discussed analysis user behavior on online game mobile from four aspect that is activeness, diurnal variation, account distribution and version distribution. A number of studies set the puzzle as a potential activity for "spatial training" in toddlers and pre-school children. For example, a cross-sectional puzzle study by Verdineetal found a high correlation between puzzle performance and various spatial skills in primary schoolaged children. Performance on standard jigsaw puzzles associated with mental rotation, spatial perception, and spatial visualization [9].

Based on the background, this study aims to analyze user behavior during playing Tangram Puzzle. The research question are (1) is there a dominant first puzzle piece selected and why it happens, then (2) is there a correlation between time and the number of completions by the player to know the perceptual speed of the player.

\section{Tangram Puzzle}

Tangram Puzzle is an old puzzle game from China. In general how to play Tangram Puzzle is the same as any other type of puzzle, that arranges each puzzle pieces into a whole picture. The system of this game is drag and drop. In Tangram Puzzle consists of 3 levels that have different levels of difficulty based on the number of puzzle pieces to be completed and time specified to finish the puzzle. Tangram puzzle interface will be shown in Figure 1. At level 1 consists of 4 puzzle pieces in 60 seconds, level 2 consists of 7 puzzle pieces in 120 seconds and level 3 consists of 9 puzzle pieces 180 seconds.

On this game page, puzzle pieces are on the right and left of the screen. The player can rotate the pieces by tapping once, then it will rotate $90^{\circ}$. The player must arrange the pieces according to the image in the middle of the screen. On the top left page, there 
is information about time and score that player gets. If a player has difficulty to solve it, there is a hint button at the top of the game page to give a clue.

Tangram Puzzle is played from the easy to hard level. Players must coherently play it. If the player can not finish the first level then he/she must repeat the level in order to continue to the next level.

\section{$3 \quad$ Methods and Data}

The focus of this research is to analyze log data from the result of playing Tangram Puzzle. This game was played by 10 people at random regardless of the environment around the player when playing. Each player plays Tangram Puzzle no time limit. Data from this research were collected based on log data of player during drag and drop puzzle pieces to complete it. Log data saved into a database. The data consists of id_user, id_scene, answer, activity, status, duration and time. Field "id_scene" to know the sequence of the pieces being worked on. For field "answer" contain the id of puzzle pieces that are drag and drop by the player. Field "status" informs about status right or the wrong player placing the puzzle pieces. Whereas the field "time" is used to record the time spent by the player to drag and drop. An example of a data log is described in Figure 2.

In the first analysis is to know the dominant first puzzle pieces selected by the player obtained based on the player sequence completing the Tangram. Then analyzed it from the shape or size, so it can be known the reason why the player tends or dominantly chooses it. And for the second analysis is using the Spearman Correlation Test that is evaluation test conducted to know the level of closeness of correlation between variables in this research. The variables are the number of drag and drop and the time spent by the player.

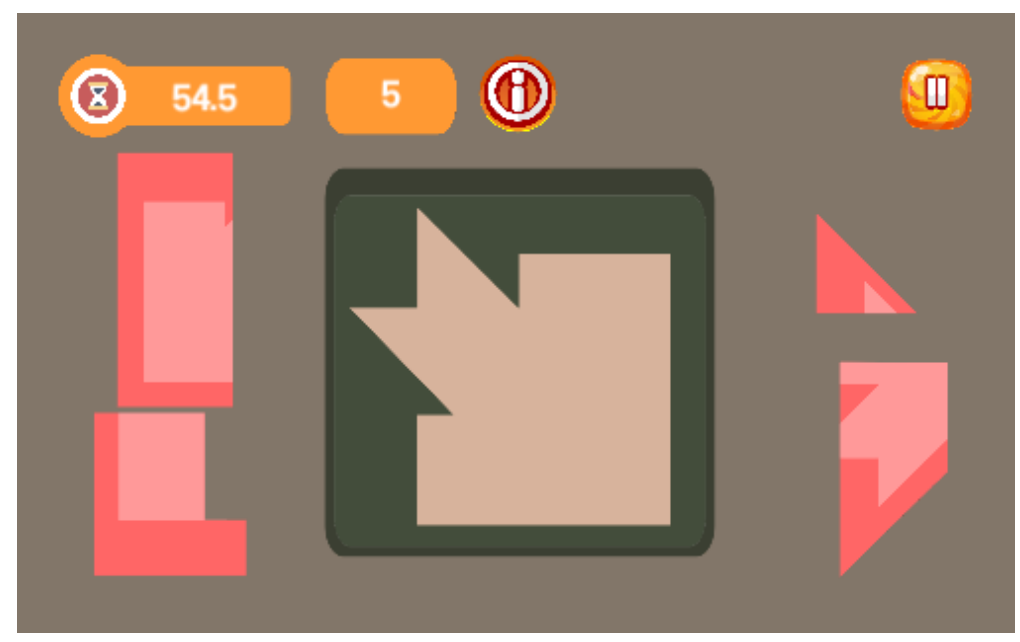

Fig. 1. Tangram Puzzle Interface 


\begin{tabular}{|r|r|r|l|l|r|l|}
\hline id_user & id_scene & answer & activity & status & duration & time \\
\hline 8 & 15 & 0 & & & 0 & $10: 51: 53$ \\
\hline 8 & 15 & 153 & DragDrop & False & 3 & $10: 51: 58$ \\
\hline 8 & 15 & 152 & DragDrop & True & 0 & $10: 52: 09$ \\
\hline 8 & 15 & 154 & DragDrop & False & 0 & $10: 52: 11$ \\
\hline 8 & 15 & 154 & DragDrop & False & 0 & $10: 52: 14$ \\
\hline 8 & 15 & 151 & DragDrop & False & 0 & $10: 52: 16$ \\
\hline 8 & 15 & 153 & DragDrop & False & 1 & $10: 52: 19$ \\
\hline 8 & 15 & 153 & DragDrop & False & 1 & $10: 52: 23$ \\
\hline 8 & 15 & 153 & DragDrop & True & 0 & $10: 52: 36$ \\
\hline 8 & 15 & 154 & DragDrop & True & 1 & $10: 52: 40$ \\
\hline 8 & 15 & 151 & DragDrop & True & 0 & $10: 52: 44$ \\
\hline
\end{tabular}

Fig. 2. Sample Log Data

Basic Decision Making in Spearman Correlation Test:

1. If the value of sig $<0.05$ then it can be concluded that there is a significant correlation between variables connected.

2. Conversely, if sig $>0.05$ then there is no significant correlation between the variables connected.

As for the level of correlation between variables ranging from \pm 0.00 to \pm 1.00 with criteria:

1. 0.00 to 0.20 means that there is almost no correlation

2. 0.21 to 0.40 means low correlation

3. 0.41 to 0.60 means medium correlation

4. 0.60 to 0.80 means high correlation

0.81 to 1.00 means perfect correlation

\section{$4 \quad$ Result and Discussion}

In this research analyze the user activity at level 1 . The purpose of this study is to answer the research question as mentioned in the introduction. (RQ1) Is there a dominant first puzzle piece selected? Why is that? Then (RQ2) Is there a correlation between time and the number of completions by the player to know the perceptual speed of the player?

\subsection{Is there a dominant first puzzle piece selected? Why does it happen?}

Based on data in the database, it can be known the sequence of players in selecting puzzle pieces. Table 1 shows the sequence of each player plays on level 1 and consist of 5 puzzles. 
Table 1. Sequence of tangram completion

\begin{tabular}{|c|c|c|c|c|c|}
\hline \multirow{2}{*}{ User } & \multicolumn{5}{|c|}{ Sequence } \\
\cline { 2 - 6 } & Puzzle 1 & Puzzle 2 & Puzzle 3 & Puzzle 4 & Puzzle 5 \\
\hline $\mathbf{1}$ & $2-4-1-2-3-1$ & $4-2-1-3-2$ & $4-1-3-2$ & $2-1-2-1-4-2-3$ & $2-3-4-1$ \\
\hline $\mathbf{2}$ & $2-4-1-3-2$ & $2-4-2-1-2-3-2$ & $2-4-1-2-3$ & $2-1-4-3$ & $2-1-3-4-1$ \\
\hline $\mathbf{3}$ & $2-3-4-1$ & $4-1-3-2$ & $4-1-3-2$ & $2-4-1-4-3$ & $2-4-1-3-4$ \\
\hline $\mathbf{4}$ & $2-4-3-4-3-1$ & $3-4-2-1$ & $4-2-1-2-3-2$ & $2-3-4-1$ & $3-2-4-1-3-4-1$ \\
\hline $\mathbf{5}$ & $2-4-3-1$ & $3-4-1-2-3$ & $4-1-2-3-1$ & $2-1-2-3-1-4$ & $2-3-1-4$ \\
\hline $\mathbf{6}$ & $2-3-1-4$ & $2-4-3-2-1$ & $4-1-2-3$ & $2-1-4-3$ & $2-4-1-3$ \\
\hline $\mathbf{7}$ & $4-2-4-3-4-1$ & $1-4-1-3-1$ & $1-4-2-3$ & $1-4-2-3$ & $3-4-1-2$ \\
\hline $\mathbf{8}$ & $2-3-1-4$ & $4-1-3-2$ & $4-2-1-3-2$ & $2-3-1-4$ & $3-4-1-2$ \\
\hline $\mathbf{9}$ & $2-4-3-1$ & $4-3-2-1-3-2$ & $4-2-1-3-2$ & $2-1-4-3$ & $3-4-2-4-1$ \\
\hline $\mathbf{1 0}$ & $3-4-1-2$ & $4-2-3-1$ & $1-4-2-3$ & $1-4-2-3$ & - \\
\hline
\end{tabular}
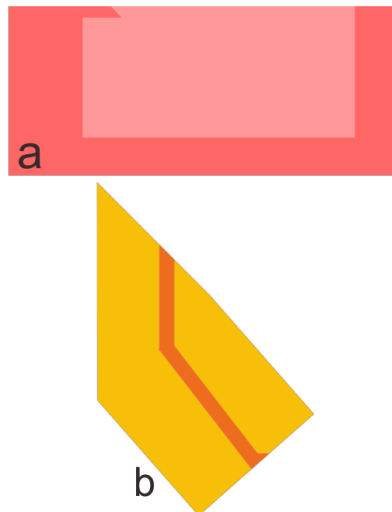
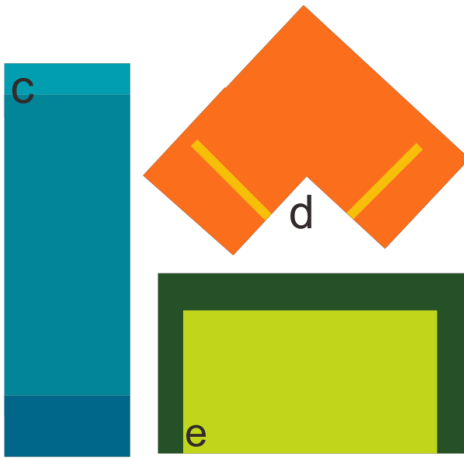

Fig. 3. Dominant puzzle pieces a. puzzle 1, b. puzzle 2, c. puzzle 3, d. puzzle 4 and e. puzzle 5

In Table 1 it can be seen that each puzzle always has the dominant puzzle pieces or the most frequently first selected by the player to finish Tangram. The five shapes of puzzle pieces have almost the same characteristics which are has a larger size than other puzzle pieces. And three of the five pieces are rectangles that are puzzle 1, 3 and 5 as shown in Figure 3. Based on these, the player will choose the largest size puzzle pieces and simple shape when playing Tangram because it will be easy to imagine the right location of the puzzle.

\subsection{Is there a correlation between time and number of completions by the} player to know the perceptual speed of the player?

By using the Spearman Correlation Test for knowing the correlation between the number of drag and drop and time spent by the player. The result will be shown in Table 2. 
Table 2. Result of Spearman Correlation Test

\begin{tabular}{|c|c|c|}
\hline Puzzle & Spearman Correlation & sig \\
\hline $\mathbf{1}$ & 0.755 & 0.012 \\
\hline $\mathbf{2}$ & 0.593 & 0.05 \\
\hline $\mathbf{3}$ & 0.564 & 0.09 \\
\hline $\mathbf{4}$ & 0.681 & 0.03 \\
\hline $\mathbf{5}$ & 0.453 & 0.221 \\
\hline
\end{tabular}

a. Puzzle 1

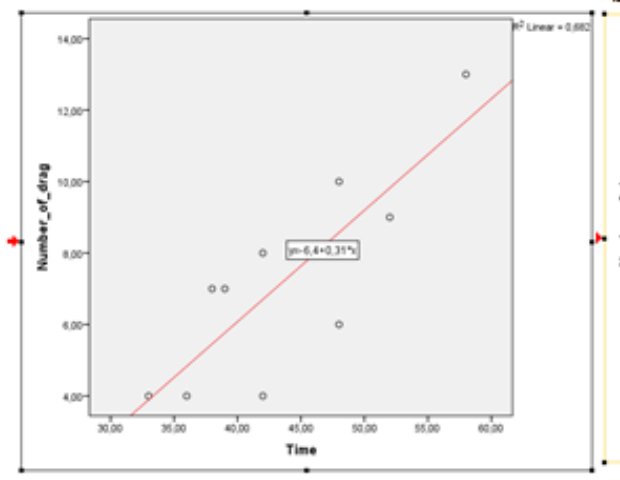

b. Puzzle 4

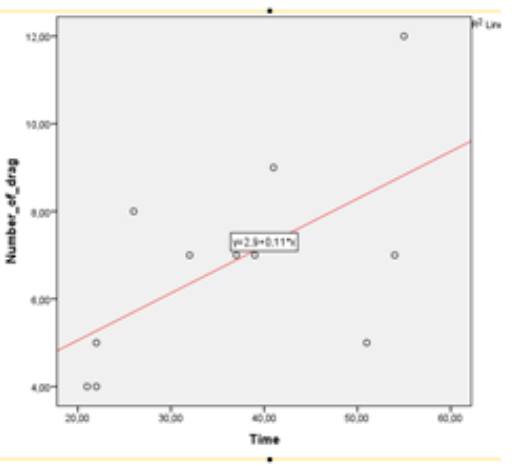

Fig. 4. Scatter plot correlation

Based on the basic decision of the Spearman Correlation Test with the value of sig ( 2 tailed) is 0.05 . It can be concluded that 3 of the 5 puzzles have a significant correlation between time and the number of completions. And from the output, Correlation Coefficient puzzle 1 and 4 have a high correlation, whereas for puzzles 2 , 3 and 5 have a moderate correlation between time and the number of completions. A positive sign indicates that the correlation between the number of drag and time is a proportionate correlation. The meaning is if the player does more drag and drop, so the time spent to complete Tangram is longer. It can be seen in Figure 4 that describes the pattern between time and the number of drag on puzzle 1 and 4 . So it can be concluded that the correlation between completions and time is moderate, quite significant, and unidirectional.

\section{Conclusion}

This research conducted user behavior analysis based on log data during play Tangram Puzzle. Based on the results of the first analysis, it can be concluded that player selects for the first shape depending on the size and the shape that is easily identified. Perceptual speed is the ability to quickly compare or recognize figures or to carry out other simple tasks involving visual perception. Based on the explanation above, it can be interpreted that the number of completions is quite influential to the time spent. It can be caused by this research conducted without regard to the 
environment around the players. So there is a possibility that disturbance from the surrounding environment. It can be concluded that when the player does a lot of drag and need a long time, not necessarily the player has less perceptual speed ability.

\section{References}

[1] Rahmawati, "Seminar Analisis Hasil TIMSS 2015," 2016, pp. 1-10, 2016.

[2] Puspendik, "Trends in International Mathematics and Science Study, Result From TIMSS 2015," Pus. Penilai. Pendidik. Badan Penelit. dan Pengemb., pp. 1-2, 2016.

[3] W. D. Prakoso, "Peningkatan Kemampuan Spasial Matematis Melalui Pembelajaran Geometri Berbantuan Geogebra," pp. 497-504, 2015.

[4] Y. T. Liao, C. H. Yu, and C. C. Wu, "Learning geometry with augmented reality to enhance spatial ability," Proc. - 2015 Int. Conf. Learn. Teach. Comput. Eng. LaTiCE 2015, pp. $221-$ 222, 2015. https://doi.org/10.1109/LaTiCE.2015.40

[5] J. Lee, "Detection and Pose Estimation of Colored Tangram Pieces : An Initial Result based on Mathematica Implementation *," no. 1, 2017.

[6] H. B. Yilmaz, "On the development and measurement of spatial ability," Int. Electron. J. Elem. Educ., vol. 1, no. 2, pp. 83-96, 2009.

[7] T. Hirasima, "Model-Based Approach for Educational Big Data Analysis of Learners Thinking with Process Data," pp. 11-16, 2017.

[8] Y. Xiong, J. Liu, Z. Lei, and L. Chen, "Traffic and User Behavior Analysis of Online Mobile Games," 2015 7th Int. Conf. Intell. Human-Machine Syst. Cybern., no. 2, pp. 117-121, 2015. https://doi.org/10.1109/IHMSC.2015.70

[9] B. N. Verdine, R. M. Golinkoff, K. Hirsh-Pasek, and N. S. Newcombe, "Finding the missing piece: Blocks, puzzles, and shapes fuel school readiness," Trends Neurosci. Educ., vol. 3, no. 1, pp. 7-13, 2014. https://doi.org/10.1016/j.tine.2014.02.005

\section{$7 \quad$ Authors}

Ivenulut Rizki Diaz Renavitasari is a Magister Student in Faculty of Computer Science, Brawijaya University, Malang. She is also a member of Multimedia, Games, and Mobile Technology Research Group. (email: ivenulutrizki@gmail.com)

Ahmad Afif Supianto is a Lecturer in Faculty of Computer Science, Brawijaya University, Malang. He is also a Coordinator of Technology-Enhanced Learning Research Group (email: afif.supianto@ub.ac.id)

Herman Tolle is a Lecturer in Faculty of Computer Science, Brawijaya University. $\mathrm{He}$ is also a Coordinator of Multimedia, Games, and Mobile Technology Research Group. (email: emang@ub.ac.id)

Article submitted 25 July 2018. Final acceptance 13 October 2018. Final version published as submitted by the authors. 